\title{
Acoustical properties of double porosity granular materials
}

\author{
Rodolfo Venegas ${ }^{\text {a) }}$ and Olga Umnova \\ Acoustics Research Centre, University of Salford, Salford M5 4WT, England
}

(Received 14 April 2011; revised 25 August 2011; accepted 11 September 2011)

\begin{abstract}
Granular materials have been conventionally used for acoustic treatment due to their sound absorptive and sound insulating properties. An emerging field is the study of the acoustical properties of multiscale porous materials. An example of these is a granular material in which the particles are porous. In this paper, analytical and hybrid analytical-numerical models describing the acoustical properties of these materials are introduced. Image processing techniques have been employed to estimate characteristic dimensions of the materials. The model predictions are compared with measurements on expanded perlite and activated carbon showing satisfactory agreement. It is concluded that a double porosity granular material exhibits greater low-frequency sound absorption at reduced weight compared to a solid-grain granular material with similar mesoscopic characteristics.

(C) 2011 Acoustical Society of America. [DOI: 10.1121/1.3644915]
\end{abstract}

PACS number(s): 43.55.Ev, 43.20.Bi, 43.20.Gp [KVH]

Pages: 2765-2776

\section{INTRODUCTION}

Properties of granular materials are of great importance in many areas of acoustics and noise control. This paper is focused on the acoustical properties of double porosity granular materials, i.e., packings of porous particles. The study of the long-wavelength sound propagation in porous media has mainly been focused on estimating two intrinsic quantities, i.e., the characteristic impedance and the wave number. ${ }^{1}$ Biot $^{2}$ investigated the problem of elastic wave propagation in a statistically isotropic porous elastic solid saturated by a viscous fluid and found that two dilatational waves, a slow and a fast wave, and one rotational wave can propagate in the medium. In this paper, only the slow wave is considered as the solid frame is assumed to be motionless. A widely accepted semiphenomenological model for the acoustical properties of materials with a single pore size or narrow pore/grain size distribution, i.e., single porosity materials, has been proposed by Johnson et al. $^{3}$ and Lafarge et al., ${ }^{4}$ and improved by Pride et $a .^{5}$ and Lafarge. ${ }^{6}$ These models make use of scaling functions that correctly match the low- and high frequency limits of the dynamic viscous $^{7}$ and thermal ${ }^{4}$ permeabilities, and rely on independently measurable macroscopic parameters; namely, porosity, static viscous and thermal permeabilities, static viscous and thermal tortuosities, tortuosity, and viscous and thermal characteristic lengths. Allard et al. ${ }^{8}$ have measured most of these macroscopic parameters for a random packing of beads and found good agreement between the model prediction and measured data. Asdrubali and Horoshenkov ${ }^{9}$ have used a rational Padé approximation to model sound propagation through expanded clay granulate. This modeling approach assumes that the material possesses capillary pores whose size distribution is log-normal. However, the pore size distribution of expanded clay may be better described as multimodal. The main drawback of these approaches is the difficulty in measuring the model parameters. Moreover, they provide

\footnotetext{
a) Author to whom correspondence should be addressed. Electronic mail: r.g.venegascastillo@edu.salford.ac.uk
}

little information about the microstructure influence on the acoustical properties. In an attempt to overcome these difficulties and provide practical expressions to work with, empirical models have been proposed by Voronina and Horoshenkov. ${ }^{10}$ They have measured flow resistivity, porosity, and tortuosity and fitted the so-called structural characteristic to predict sound propagation in granular materials such as perlite, vermiculite, and granulate nitrile. An empirical model has, however, limited predictive power and does not provide an understanding of the material morphology influence on the acoustical properties. A microstructure-based approach overcomes these problems. It requires information about the material structure such as particle shapes, sizes, and arrangement and relies on solving the oscillatory fluid flow and heat conduction problems in a representative geometry. Chapman and Higdon $^{11}$ have calculated dynamic viscous permeability of monodisperse arrays of spheres arranged in periodic lattices. Umnova et al. ${ }^{12}$ have extended the "cell model" approach to calculating the drag parameters of packing of spheres considering a geometrically justified outer cell radius. These authors have also provided expressions for dynamic bulk modulus based on a mathematical similarity between the oscillatory fluid flow and heat conduction problems. ${ }^{13}$

The homogenization of periodic media theory ${ }^{14,15}$ (HPM) has been applied by Gasser et al. ${ }^{16}$ to numerically solve the boundary value problems at different scales and calculate both the macroscopic material parameters and the acoustical properties of a face-centered cubic (fcc) packing of spheres. A similar work, based on numerical HPM, has been published by Lee et al. ${ }^{17}$ for simple cubic, bodycentered cubic (bcc), fcc, and hexagonal close-packed arrays. Boutin and Geindreau ${ }^{18}$ have proposed analytical estimates of dynamic viscous permeabilities for granular media using a combined HPM and self-consistent approach. The description of the acoustical properties of granular materials is completed with an analytical expression for dynamic thermal permeability also derived by these authors. ${ }^{19}$

An emerging field is the study of the acoustical properties of double porosity materials which are efficient at absorbing 
low frequency sound. In these materials, two fluid networks with well-separated characteristic pore or inclusion sizes can be identified. The equations for sound propagation in double porosity materials have been derived by Auriault and Boutin ${ }^{20}$ and Boutin et al. ${ }^{21}$ using HPM. It has been pointed out in a later work $^{22}$ that the material properties strongly depend on the ratio $\varepsilon_{0}$ between the characteristic size of the microscopic scale $l_{m}$ and that of mesoscopic scale $l_{p}$. At moderate interscale ratio values, e.g., $\varepsilon_{0}=l_{m} / l_{p} \approx 10^{-1}$, the two fluid networks strongly interact and influence both the macroscopic fluid flow and heat conduction. This case is usually referred to as low-permeability contrast. Materials with this property have been experimentally and theoretically investigated by Pispola et al. ${ }^{23}$ For small interscale ratio values, e.g., $\varepsilon_{0} \approx 10^{-3}$, the macroscopic flow is determined by the mesoscopic fluid network. The dynamic bulk modulus is modified due to pressure diffusion effects in the microdomain. This leads to an additional dissipation term which depends on the mesoscale geometry and the microdomain characteristics. This effect is specific to double porosity materials and has been experimentally confirmed for perforated porous panels ${ }^{24}$ and for porous materials with porous inclusions. ${ }^{25}$

In this paper, the acoustical properties of double porosity granular materials, i.e., packings of porous particles, are investigated both theoretically and experimentally. The paper is organized as follows. In Sec. I, the wave equation describing sound propagation in double porosity materials of arbitrary geometry is presented. The calculations of the dynamic density and bulk modulus are outlined. In Sec. II the general theory is applied to packings of identical porous spheres. Experimental validation of the models and discussion are presented in Sec. III. Measurements on both lowand high-permeability contrast granular materials (expanded perlite and activated carbon, respectively) are reported as well as the methods used to estimate their structural characteristics. The main findings are summarized in Sec. IV.

\section{THEORY}

\section{A. Sound propagation in double porosity materials-Overview}

Following Refs. 20-22, three scales can be identified and used to describe sound propagation in a rigid-frame double porosity granular material saturated by a Newtonian fluid, e.g., air. They are schematically shown in Fig. 1. The macroscopic characteristic size $L$ is associated with the sound wavelength $\lambda$ in the material as ${ }^{22} L=O(|\lambda / 2 \pi|)$. The characteristic size $l_{p}$ is determined by the size of the mesoheterogenities, which in the case under study corresponds to the particle size. The characteristic size $l_{m}$ is determined by the size of the pores within the particle. This characteristic size is assumed large enough so that the saturating fluid is continuously distributed throughout the space it occupies. Rarefaction effects are therefore neglected. However, it has been shown that these effects do not change the form of the macroscopic isothermal acoustic description in single ${ }^{26}$ and double ${ }^{27}$ porosity materials but the way the effective quantities are calculated. To model the material as a homogenous equivalent fluid, the separation of scales assumption should

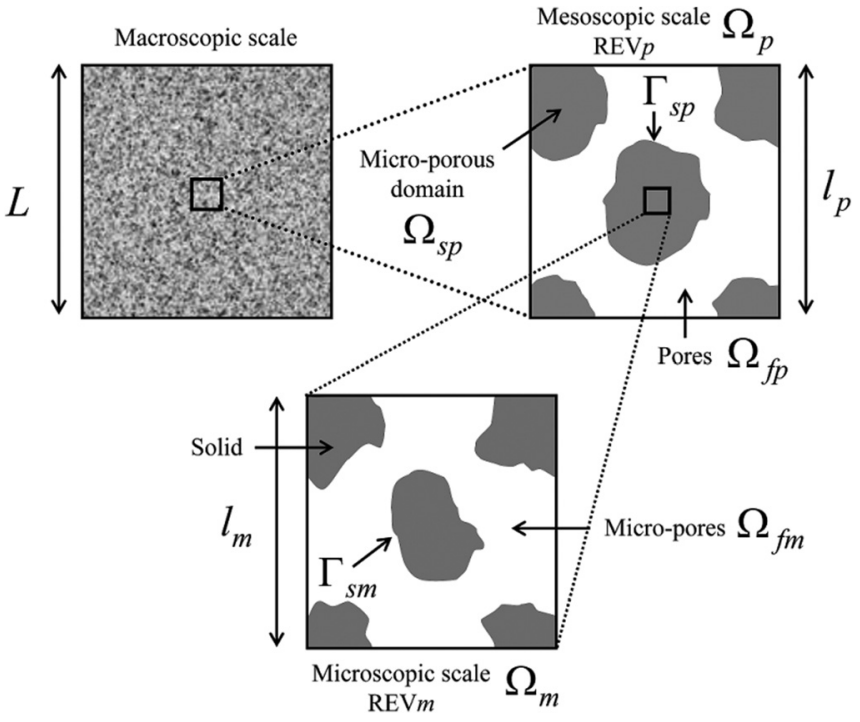

FIG. 1. Three scales of the double porosity material (adapted from Ref. 22).

be satisfied, e.g., $\varepsilon=l_{p} / L \ll 1$ and $\varepsilon_{0}=l_{m} / l_{p} \ll 1$. The existence of the representative elementary volumes $\operatorname{REVm} \Omega_{m}$ and $\operatorname{REVp} \Omega_{p}$ can then be ensured provided that the conditions on the interscale ratios are fulfilled. The mesoscale and microscale porosities are $\phi_{p}=\Omega_{f p} / \Omega_{p}$ and $\phi_{m}=\Omega_{f m} / \Omega_{m}$, respectively, where $\Omega_{f i}$ is the open voids/pore volume and $\Omega_{i}$ is the volume of the REVs. Here and in the following $i=p, m$. The overall porosity of the material is given by $\phi_{d b}=\phi_{p}+\left(1-\phi_{p}\right) \phi_{m}$. The subscript $d b$ denotes a double porosity quantity from now on.

The wave equation for acoustic pressure $p$ in a rigidframe double porosity material is given by ${ }^{22}$

$$
\frac{j \omega p}{K_{d b}(\omega)}-\nabla \cdot\left(\frac{\mathbf{k}_{d b}(\omega)}{\eta} \nabla p\right)=0 .
$$

Time dependence in the form $e^{j \omega t}$ is assumed. Here, $\eta$ is the dynamic viscosity and $K_{d b}$ and $\mathbf{k}_{d b}$ are the dynamic bulk modulus and dynamic permeability of the double porosity material. The latter becomes a scalar quantity for isotropic media, i.e., $\mathbf{k}_{d b}=k_{d b} \mathbf{I}$, where $\mathbf{I}$ is the unitary second-rank tensor. The way of calculating $K_{d b}$ and $\mathbf{k}_{d b}$ depends on the value of the interscale ratio $\varepsilon_{0}$. When $\varepsilon_{0} \approx 10^{-3}$ a highpermeability contrast is achieved and the pores in the particles have negligible contribution to the macroscopic fluid flow. In this case, the dynamic permeability $k_{d b}$ coincides $^{22}$ with that of a packing of solid particles, i.e.,

$$
\mathbf{k}_{d b}(\omega)=\mathbf{k}_{p}(\omega)+\left(1-\phi_{p}\right) \mathbf{k}_{m}(\omega) \approx \mathbf{k}_{p}(\omega),
$$

where $\mathbf{k}_{p}$ and $\mathbf{k}_{m}$ are the dynamic permeability tensors of the mesoscopic and the microscopic domains, respectively.

The dynamic bulk modulus for a material with highpermeability contrast is given by ${ }^{22}$

$$
K_{d b}(\omega)=\left(\frac{1}{K_{p}(\omega)}+\frac{\left(1-\phi_{p}\right)}{K_{m}(\omega)} F_{d}\left(\frac{P_{0}}{\phi_{m}} \frac{\omega}{K_{m}(\omega)}\right)\right)^{-1},
$$


where $K_{p}$ and $K_{m}$ are the dynamic bulk moduli of the mesoscopic and microscopic domain, respectively. These can be expressed in terms of the dynamic thermal permeabilities $k_{p}^{\prime}$ and $k_{m}^{\prime}$ as $^{4}$

$$
K_{i}=\frac{\gamma P_{0}}{\phi_{i}}\left(\gamma-j \omega \rho_{0}(\gamma-1) C_{p} \frac{k_{i}^{\prime}(\omega)}{\kappa \phi_{i}}\right)^{-1},
$$

where $\rho_{0}$ is the gas density, $C_{p}$ is the specific heat at constant pressure, $\kappa$ is the thermal conductivity, and $\gamma$ is the adiabatic constant.

In the case of low-permeability contrast, e.g., $\varepsilon_{0} \approx 10^{-1}$, the dynamic permeability of the double porosity material is given, in a first approximation, by a parallel flow model. ${ }^{22}$ Hence, the dynamic permeability of the microscopic domain $\mathbf{k}_{m}(\omega)$ in Eq. (2) cannot be neglected. This expression holds for mesoscopic geometries with straight pores or slits. ${ }^{22}$ The dynamic bulk modulus under low-permeability contrast condition is obtained by replacing the function $F_{d}$ by 1 in Eq. (3). The way of calculating the dynamic viscous and thermal permeabilities and the function $F_{d}$ is now detailed.

The fluid flow at the microscopic and mesoscopic levels is described by an oscillatory Stokes forced problem with no-slip boundary condition on $\Gamma_{s i}$. This problem is linear and can be written in terms of the $\Omega_{i}$-periodic dynamic permeability field $\widehat{\mathbf{k}}_{i}$ and the zero mean value pressure $\widehat{\pi}_{i}$ as ${ }^{7}$

$$
\begin{gathered}
j \omega \frac{\rho_{0}}{\eta} \widehat{\mathbf{k}}_{i}+\nabla \widehat{\pi}_{i}-\Delta \widehat{\mathbf{k}}_{i}=\mathbf{I} \text { in } \Omega_{f i}, \\
\nabla \cdot \widehat{\mathbf{k}}_{i}=\mathbf{0} \text { in } \Omega_{f i} \text { and } \widehat{\mathbf{k}}_{i}=\mathbf{0} \text { on } \Gamma_{s i} .
\end{gathered}
$$

The dynamic permeability is then calculated by averaging the solution over the REV $i$ as $\mathbf{k}_{i}(\omega)=\left\langle\widehat{\mathbf{k}}_{i}\right\rangle_{i}$ with $\langle\cdot\rangle_{i}=\left(1 / \Omega_{i}\right) \int_{\Omega_{f i}} \cdot d \Omega$. For isotropic microscopic media it becomes $\mathbf{k}_{i}=k_{i} \mathbf{I}$.

The thermal exchanges between the saturating fluid and the solid matrix are described by an oscillatory heat conduction problem with isothermal condition applied on $\Gamma_{s i}$. This linear problem can be written in terms of the $\Omega_{i}$-periodic dynamic thermal permeability distribution $\widehat{k}_{i}$ as follows: ${ }^{4}$

$$
j \omega \frac{\rho_{0} C_{p}}{\kappa} \widehat{k_{i}^{\prime}}-\Delta \widehat{k_{i}^{\prime}}=I \quad \text { in } \quad \Omega_{f i} \text {, and } \widehat{k_{i}^{\prime}}=0 \text { on } \Gamma_{s i}
$$

The dynamic thermal permeabilities of the microdomains and mesodomains are then calculated by averaging the solution of Eq. (8) over the REV $i$ as $k_{i}^{\prime}(\omega)=\left\langle k_{i}^{\prime}\right\rangle_{i}$.

The function $F_{d}$ in Eq. (3) is defined as the ratio of the average pressure in the microscopic domain to that in the mesoscopic domain. ${ }^{22}$ It is related to the dynamic pressure diffusion function $D(\omega)$ as ${ }^{22}$

$$
F_{d}(\omega)=1-j \omega \eta \frac{\phi_{m}}{P_{0}} k_{0 m}^{-1} \frac{D(\omega)}{\left(1-\phi_{p}\right)}
$$

where $k_{0 m}$ is static viscous permeability of the microdomain and is calculated from the solution of Eqs. (5), (6) for $\omega=0$. In this expression, the material has been assumed isotropic or just the preferential flow direction is taken into account.
In addition, the sound propagation in the microscopic domain has been assumed isothermal [this also implies replacing $K_{m}(\omega)$ by $P_{0} / \phi_{m}$ in Eq. (3)], and in a viscous regime. The pressure in the microscopic domain is not uniform and governed by a diffusion equation with boundary condition imposed by pressure in the mesoscopic domain. ${ }^{22}$ This problem can be also formulated in terms of the $\Omega_{p}$-periodic dynamic pressure diffusion distribution $\widehat{D}$ as ${ }^{22}$

$$
\Delta \widehat{D}-j \omega \eta \frac{\phi_{m}}{P_{0}} k_{0 m}^{-1} \widehat{D}=-1 \text { in } \Omega_{s p} \text { and } \widehat{D}=0 \text { on } \Gamma_{s p} .
$$

Pressure diffusion function $D(\omega)$ is then calculated by averaging the solution over the solid phase of the REVp as

$$
D(\omega)=\langle\widehat{D}\rangle=\frac{1}{\Omega_{p}} \int_{\Omega_{s p}} \widehat{D} d \Omega
$$

This completes the direct approach. All the quantities can be calculated from their definitions for every frequency of interest. This could, however, require a significant amount of computation time when dealing with realistic geometries. To overcome this problem, one can use scaling functions that correctly match the low- and high-frequency asymptotics of the dynamic permeabilities, ${ }^{5}$ bulk moduli, ${ }^{6}$ and pressure diffusion function. ${ }^{28}$ In this way, the acoustic description is reduced to solving three static problems per scale and one for the pressure diffusion function. The form of the scaling functions is the same for all of the abovementioned quantities and is the following:

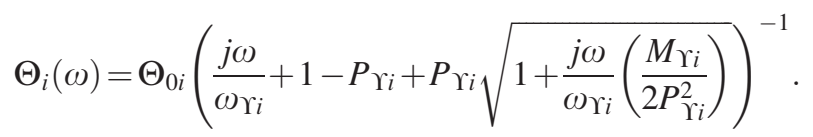

The symbol $\Theta$ is used to represent viscous, thermal or pressure diffusion quantities and the subscript $\Upsilon$ indexes the characteristic frequencies $\omega_{\Upsilon i}$ and shape factors $M_{\Upsilon_{i}}$ and $P_{\Upsilon_{i}}$ accordingly. For viscosity related quantities $\Theta_{i}=k_{i}$ and $\Upsilon=v$ with $P_{v i}=M_{v i} / 4\left(\alpha_{0 i} / \alpha_{\infty i}-1\right), M_{v i}=8 k_{0 i} \alpha_{\infty i} / \phi_{i} \Lambda_{i}^{2}$, and the viscous characteristic frequency defined as $\omega_{v i}=\eta \phi_{i} / k_{0 i} \alpha_{\infty i} \rho_{0}$. For temperature related quantities $\Theta_{i}=k_{i}^{\prime} \quad$ and $\Upsilon=t \quad$ with $\quad P_{t i}=M_{t i} / 4\left(\alpha_{0 i}^{\prime}-1\right)$, $M_{t i}=8 k_{0 i}^{\prime} / \phi_{i} \Lambda_{i^{2}}^{\prime}$, and the thermal characteristic frequency defined as $\omega_{t i}=\kappa \phi_{i} / C_{p} \rho_{0} k_{0 i}^{\prime}$. In these expressions, $k_{0 i}$ and $k_{0 i}^{\prime}$ are the static viscous and thermal permeabilities. The static viscous and thermal tortuosities are calculated as $\alpha_{0 i}=\phi_{i}\left\langle\widehat{k}_{0 i}^{2}\right\rangle_{i}\left\langle\hat{k}_{0 i}\right\rangle_{i}^{-2}$ and $\alpha_{0 i}^{\prime}=\phi_{i}\left\langle\widehat{k^{\prime 2}}\right\rangle_{i}\left\langle\widehat{k^{\prime}}{ }_{0 i}\right\rangle_{i}^{-2}$. High frequency tortuosities and viscous characteristic lengths for isotropic materials are obtained from ${ }^{3,29} \phi_{i} \alpha_{\infty i}^{-1}=\left\langle\mathbf{E}_{i} \cdot \mathbf{e}\right\rangle_{i}$ and $\Lambda_{i}=2 \int_{\Omega_{f i}} \mathbf{E}_{i} \cdot \mathbf{E}_{i} d \Omega / \int_{\Gamma_{s i}} \mathbf{E}_{i} \cdot \mathbf{E}_{i} d \Gamma$, where the scaled electric field (local electrical field divided by the applied macroscopic potential gradient) is given by $\mathbf{E}_{i}=\mathbf{e}-\nabla \vartheta_{i}$ and $\vartheta_{i}$ is the $\Omega_{i}$-periodic deviatoric part of an electric potential. This can be calculated from the solution of ${ }^{29}$ 


$$
\Delta \vartheta_{i}=0 \text { in } \Omega_{f i} \text { and } \mathbf{n} \cdot \nabla \vartheta_{i}=\mathbf{n} \cdot \mathbf{e} \text { on } \Gamma_{s i} .
$$

The thermal characteristic length is a geometrical parameterdefined as twice the volume-to-pore-surface ratio, ${ }^{30}$ i.e., $\Lambda_{i}^{\prime}=2 \Omega_{f i} / \Gamma_{s i}$. For pressure diffusion related quantities $\Theta_{i}=D$ and $\Upsilon_{i}=d$ with $P_{d}=M_{d} / 4\left(\alpha_{d}-1\right), M_{d}=8 D_{0} /$ $\left(1-\phi_{p}\right) \Lambda_{d}^{2}$, and the pressure diffusion characteristic frequency defined as $\omega_{d}=\left(1-\phi_{p}\right) P_{0} k_{0 m} / \eta \phi_{m} D_{0}$. The static pressure diffusion parameter $D_{0}$ is calculated from the solution of Eq. (9) for $\omega=0$. The static pressure diffusion tortuosity is defined as ${ }^{28} \alpha_{d}=\left(1-\phi_{p}\right)\left\langle\widehat{D_{0}^{2}}\right\rangle\left\langle\widehat{D}_{0}\right\rangle^{-2}$ and the pressure diffusion characteristic length as ${ }^{22} \Lambda_{d}=2 \Omega_{s p} / \Gamma_{s p}$.

The characteristic impedance and the wave number are related to the dynamic density, $\rho_{d b}(\omega)=\eta k_{d b}^{-1}(\omega) / j \omega$, and dynamic bulk modulus as ${ }^{1}$ $Z_{d b}^{c}(\omega)=\sqrt{\rho_{d b} K_{d b}}$ and $q_{d b}^{c}(\omega)=\omega \sqrt{\rho_{d b} K_{d b}^{-1}}$. Surface impedance of a rigidly-backed material layer of thickness $d$ is then calculated as ${ }^{1} Z_{d b}^{w}(\omega)=-j Z_{d b}^{c} \cot \left(q_{d b}^{c} d\right)$. Pressure reflection coefficient $R_{d b}$ and normal incidence sound absorption coefficient $\alpha_{d b}$ are given by ${ }^{1}$

$$
R_{d b}(\omega)=\frac{Z_{d b}^{w}-Z_{0}}{Z_{d b}^{w}+Z_{0}} \quad \text { and } \quad \alpha_{d b}(\omega)=1-\left|R_{d b}\right|^{2}
$$

where $Z_{0}$ is the characteristic impedance of air.

In summary, dynamic viscous and thermal permeabilities of the microdomain and mesodomain are needed to model the acoustical properties of a double porosity lowpermeability contrast material. To model materials with high-permeability contrast dynamic viscous and thermal permeabilities for the mesodomain, the static values of the bulk modulus and viscous permeability of the microdomain and the pressure diffusion function are required. The analytical expressions used for modeling double porosity granular materials are presented in the next section.

\section{B. Analytical model for double porosity granular materials}

In a first approximation, an unconsolidated double porosity granular material can be modeled as an array of identical porous spheres. The pores in the spheres can be assumed cylindrical. The mesoscopic domain is characterized by the particle radius $r_{p}$ and the intergranular porosity $\phi_{p}=\Omega_{f p} / \Omega_{p}$, while the pore radius $r_{m}$ and the microporosity $\phi_{m}=\Omega_{f m} / \Omega_{m}$ characterize the microscopic domain.

First, dynamic viscous and thermal permeabilities of the mesodomain are calculated. Different estimates for dynamic viscous permeability of single porosity granular materials have been deduced using HPM combined with a selfconsistent approach. ${ }^{18}$ The inclusion composed of a concentrically arranged solid particle in a fluid shell is assumed to be surrounded by a Darcy medium. The oscillatory flow in the fluid shell is described by a Stokes forced problem. A boundary condition of zero velocity is applied on the particle surface. At the inclusion boundary, the normal velocity components in the fluid and in the equivalent Darcy medium are assumed equal. Following energy consistency arguments, two possible conditions can be imposed on the stress on the inclusion boundary. For "pressure" or static approach (P-estimate) the normal stress component in the fluid matches the pressure in the equivalent Darcy medium. In this case, the tangential velocity component does not match that in the Darcy medium, as is the case for the "velocity" or kinematic approach (V-estimate). The P-estimate is considered here because it is energy consistent, correct up to the first order (in terms of the expansion parameter $\varepsilon$ ) compared to the macroscopic description obtained using HPM, and provides closer agreement with numerical results for regular packing of spheres. ${ }^{18}$ It results in the following expression for dynamic viscous permeability:

$$
k_{p}(\omega)=\frac{\delta_{v}^{2}}{\left(1-\frac{3 C}{x^{2}}\right)},
$$

where

$$
\begin{aligned}
& C=\frac{A x+B \tanh (x(\beta-1))}{a x+b \tanh (x(\beta-1))} \\
& A=\left(3+(\beta x)^{2}\right)\left(1+\frac{x^{2}}{6}\right)-3 \beta\left(1+\frac{x^{2}}{2}\right) \\
& B=\left(3+(\beta x)^{2}\right)\left(1+\frac{x^{2}}{2}\right)-3 \beta x^{2}\left(1+\frac{x^{2}}{6}\right) \\
& a=\frac{1}{3}\left(3+(\beta x)^{2}\right)-3 \beta-\frac{2}{\beta}\left(1+\frac{x^{2}}{6}\right)+\frac{4}{\cosh (x(\beta-1))} \\
& b=3+\beta(\beta-1) x^{2}-\frac{2}{\beta}\left(1+\frac{x^{2}}{2}\right), \\
& \delta_{v}=\sqrt{\frac{\eta}{j \rho_{0} \omega}}, \quad \beta=\left(1-\phi_{p}\right)^{1 / 3}, \quad \text { and } x=\frac{r_{p}}{\beta \delta_{v}} .
\end{aligned}
$$

The static viscous permeability is recovered from Eq. (14) assuming that frequency tends to zero: ${ }^{18}$

$$
k_{0 p}=\left(\frac{r_{p}^{2}}{3 \beta^{2}}\right)\left(\frac{\left(2+3 \beta^{5}\right)}{\beta\left(3+2 \beta^{5}\right)}-1\right) .
$$

The corresponding dynamic thermal permeability is calculated from the solution of an oscillatory heat conduction problem with isothermal boundary condition on the particle surface and zero temperature gradient on the inclusion boundary. ${ }^{19}$ It is given by

$$
k_{p}^{\prime}(\omega)=\delta_{t}^{2}\left(1-\beta^{3}+\frac{3 \beta}{x_{t}^{2}}\left(\beta x_{t} \frac{1+x_{t} \tanh \left(x_{t}(\beta-1)\right)}{x_{t}+\tanh \left(x_{t}(\beta-1)\right)}-1\right)\right),
$$

where $\delta_{t}=\sqrt{N_{p r}} \delta_{v}$ and $x_{t}=x / \sqrt{N_{p r}}$. The Prandlt number is defined as $N_{p r}=C_{p} \eta / \kappa$.

The expressions for dynamic viscous and thermal permeabilities of a cylindrical pore network for the microdomain 
are calculated from the solution of Eqs. (5)-(7). These are given by ${ }^{1}$

$$
\begin{aligned}
& k_{m}(\omega)=\phi_{m} \delta_{v}^{2}(1-G(s)), \text { and } \\
& k_{m}^{\prime}=\phi_{m} \delta_{t}^{2}\left(1-G\left(\sqrt{N_{p r}} s\right)\right) .
\end{aligned}
$$

where

$$
G(s)=\frac{2}{s} \frac{J_{1}(s)}{J_{0}(s)} \text { and } s=\sqrt{-j} \frac{r_{m}}{\left|\delta_{v}\right|} .
$$

Here $J_{0,1}$ are Bessel functions of the first kind. The static viscous permeability is ${ }^{1} k_{0 m}=\frac{\phi_{m} r_{m}^{2}}{8}$. The pressure diffusion function $D(\omega)$ has been analytically calculated for a packing of identical spherical porous particles as follows. Allowing only for radial diffusion, Eq. (9) is written in spherical coordinates as

$$
\frac{2}{r} \frac{\partial \widehat{D}}{\partial r}+\frac{\partial^{2} \widehat{D}}{\partial r^{2}}-j \delta_{d}^{-2} \widehat{D}=-1 \text { and } \widehat{D}\left(r=r_{p}\right)=0,
$$

where the pressure diffusion skin depth is defined as $\delta_{d}=\sqrt{\frac{P_{0} k_{0 m}}{\phi_{m} \eta \omega}}$. Multiplying Eq. (25) by $r^{2}$ and making the change of variable $\zeta=\sqrt{-j} \delta_{d}^{-1} r$, one can obtain a nonhomogenous spherical Bessel equation $\zeta^{2} \partial_{\zeta \zeta} \widehat{D}+2 \zeta \partial_{\zeta} \widehat{D}+\zeta^{2} \widehat{D}$ $=-j \delta_{d}^{2} \zeta^{2}$, with $\widehat{D}\left(\zeta=\sqrt{-j} r_{p} \delta_{p}^{-1}\right)=0$. The general solution of this equation is ${ }^{31} \widehat{D}_{h}=A j_{0}(\zeta)+B y_{0}(\zeta)-j \delta_{d}^{2}$, where $j_{0}, y_{0}$ are spherical Bessel functions of first and second kind of order 0 . The constant $B$ is set to zero as the solution cannot be infinite at the particle center. The coefficient $A$ is calculated from the boundary condition as $A=j \delta_{d}^{2} \frac{z}{\sin (z)}$, where $z=\sqrt{-j} r_{p} \delta_{d}^{-1}$. Here, the identity ${ }^{31} j_{0}(\zeta)=\sin (\zeta) / \zeta$ was used to derive this expression. The pressure diffusion function is then obtained by integrating $\widehat{D}$ over the volume of the porous sphere as $D(\omega)=\left(1-\phi_{p}\right) r_{p}^{2}\left(3-3 z \cot (z)-z^{2}\right) / z^{4}$. The function $F_{d}$ is related to $D(\omega)$ through Eq. (8) as

$$
F_{d}(\omega)=\frac{3}{z^{2}}(1-z \cot (z)) \text {. }
$$

Sound propagation in the microdomain has been assumed isothermal and in a viscous regime. In a more general case of non-isothermal and visco-inertial sound propagation, $z$ should be replaced by $z=r_{p} \omega \sqrt{\left(\phi_{m} / P_{0}\right)\left(\eta / j \omega k_{0 m}\right)}=r_{p}$ $\omega \sqrt{\rho_{m} K_{m}^{-1}}=r_{p} q_{m}^{c}(\omega)$. However, this generalization does not seem to be necessary for common acoustic materials in the audible range of frequencies.

\section{MODEL VALIDATION AND DISCUSSIONS}

To validate the models, measurements of sound absorption coefficient of double porosity materials have been conducted in a vertically installed B\&K 4206 impedance tube. The two-microphone method described in the standard ${ }^{32}$ ISO 10534-2:2001 has been used. The analytical model for single porosity granular materials is validated first by conducting measurements on a packing of lead shots. Validations for both low and high permeability contrast double porosity materials are performed after that. Expanded perlite serves to compare predictions given by a hybrid model for low-permeability contrast granular materials. The term "hybrid model" is used in this paper if at least one of the quantities is numerically calculated using scaling functions [Eq. (11)]. Two activated carbon samples are used to validate the model for double porosity high-permeability contrast granular materials. For every case, a detailed description of the methods used to estimate the characteristic sizes and porosities is presented.

\section{A. Single porosity granular material}

\section{Characterization}

The particle radius distribution of lead shots has been measured using optical granulometry. The image processing consists of adjusting the contrast, converting to black and white, and performing morphological operations to fill the holes. ${ }^{33}$ The processed image is then analyzed to obtain the equivalent radius of each particle. This has been done by fitting a circle with the same area as the pixel region. Figure 2 shows the complementary cumulative distribution function of the equivalent particle radius obtained using 108 particles. The particle radius distribution is close to normal, with a mean value of $\bar{r}_{p}=0.5507 \mathrm{~mm}$ and a standard deviation of $0.0209 \mathrm{~mm}$. Considering the non-perfect nature of the lead shots and that the nominal radius is $0.5 \mathrm{~mm}$ the agreement between this value and the expected value of the fitted distribution can be considered satisfactory. The bulk density of the packing has been measured by weighting a small cubic container of a known volume filled with the shots. Using the tabulated value of lead density $\left(\rho_{l s}=11.34 \mathrm{~g} / \mathrm{cm}^{3}\right)$ the porosity of the packing has been estimated as $\phi_{p}=1-\rho_{b} / \rho_{l s}=0.3905$, where $\rho_{b}=6.9117 \mathrm{~g} / \mathrm{cm}^{3}$ is the bulk density of the packing.

\section{Comparison with data}

The sound absorption coefficient of a lead shot layer is calculated using Eq. (13) with dynamic viscous and thermal permeabilities given by Eqs. (14) and (22). The subscript $d b$ in Eq. (13) should be replaced by $p$ to reflect the single porosity nature of the packing. Measured and predicted values of pressure reflection coefficient are shown in Figs. 3(a) and 3(b) for frequencies between 150 to $6400 \mathrm{~Hz}$. The inset plot [Fig. 3(c)] shows the sound absorption coefficient. The slight disagreement between the data and the predictions could be due to limited applicability of the analytical model for low porosity materials $\left(\phi_{p}<0.6\right)$ as suggested in Ref. 19. The inaccuracy, due to finite particle size, in defining the layer thickness could also contribute to the disagreement. If the layer thickness is set to $d=3.1652 \mathrm{~cm}$, a better agreement is obtained as is also shown in Figs. 3(a)-3(c). The model for the acoustical properties of single porosity granular materials has been therefore validated and will be further used in modeling double porosity granular materials. 


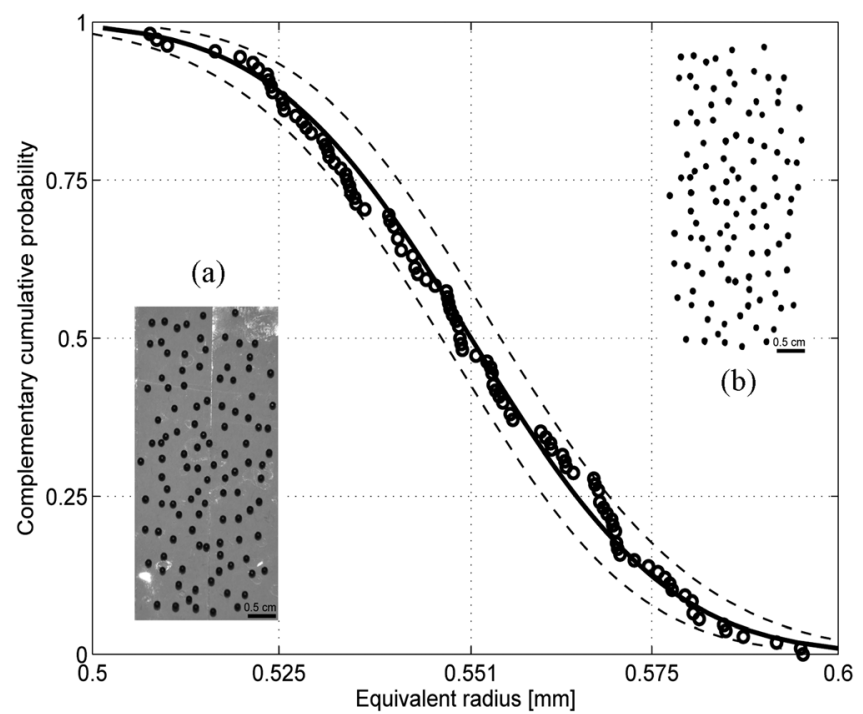

FIG. 2. Complementary cumulative distribution function for lead shot particle radius. Circles-data. Continuous line-fitted normal distribution. Dashed lines-95\% confidence interval. The inset plots show the original (a) and the processed (b) images.

\section{B. Double porosity granular material with low-permeability contrast}

\section{Characterization}

Expanded perlite is a chemically-inert industrial mineral made out of naturally occurring siliceous volcanic rock. This material is commonly used in ceiling tiles and roof insulation products. The sample used in this work is a commercial product called Expanded Perlite P3 (P3 from now on). The estimated density of the frame is $2.774 \mathrm{~g} / \mathrm{cm}^{3}$. The bulk density has been measured using the procedure described in the previous section and is equal to $0.062 \mathrm{~g} / \mathrm{cm}^{3}$. The overall porosity is therefore $\phi_{d b}=0.9776$. This value does not necessarily correlate with the "open" porosity commonly used in acoustics but includes "closed" pores as well. The fact that this value is used in calculations inevitably affects the accuracy of the predictions.

The P3 grains are fairly ellipsoidal with a nominal $80 \%$-band grain size of $0.75-1.2 \mathrm{~mm}$. This means that $10 \%$ of the grain sizes fall below or above this range. It has not been possible to apply optical granulometry to this sample due to its extremely light-weight nature. In the absence of the actual particle size distribution, the equivalent particle radius has been calculated as half of the average between the upper and lower limit of the $80 \%$-band grain size, i.e., $r_{p}=0.4875 \mathrm{~mm}$. It is known that identical nonspherical particles can pack better than spherical particles. $^{34}$ Therefore, the mesoporosity can be smaller than that for a random close packing of identical spheres, which is given by ${ }^{35} \phi_{p} \approx 0.36$. On the other hand, the effect of polydispersity is manifested through a decrease in porosity as small particles can fit in between the voids formed by larger particles. An arrangement that reflects this phenomenon is the bcc packing. The mesoporosity is therefore set equal to that of the bcc close packing, i.e., $\phi_{p}=0.32$. It is, however, recognized this value is somewhat arbitrary.

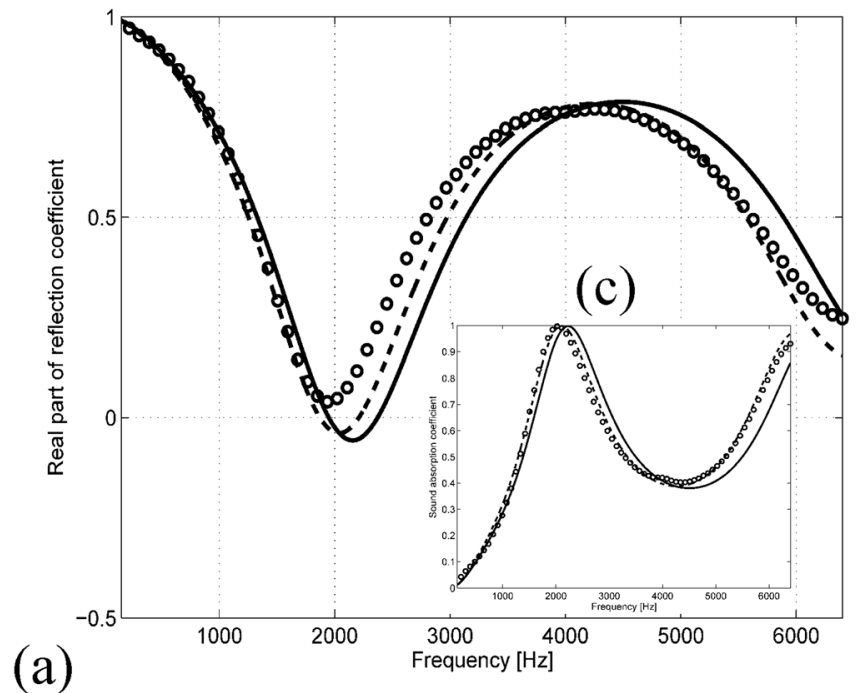

(a)

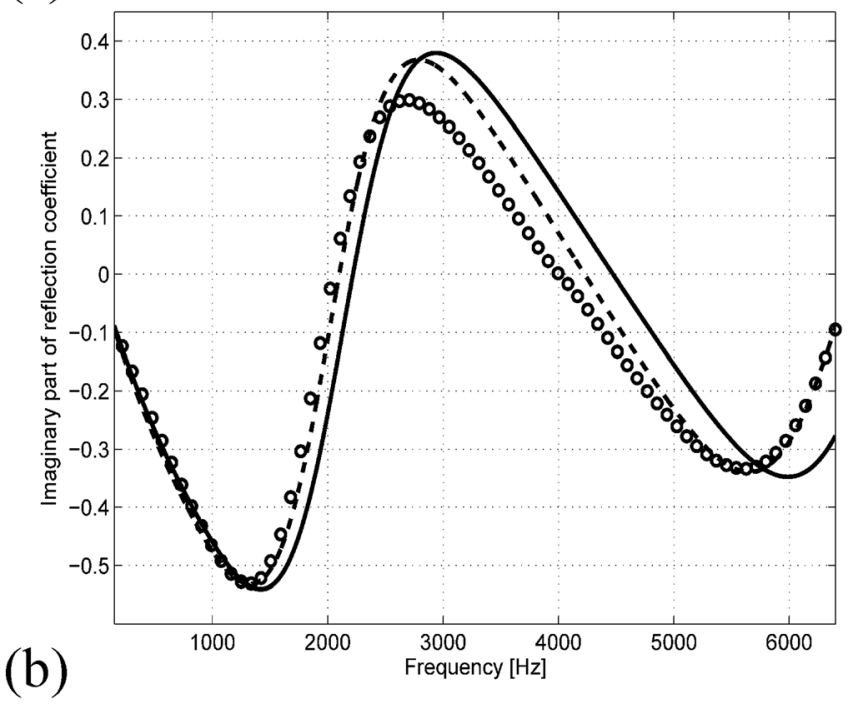

FIG. 3. Real (a) and imaginary (b) parts of the reflection coefficient of a 3 -cm hard-backed layer of lead shots. Circles-data. Continuous linepredictions. Dashed line-model predictions for a layer thickness of $3.1652 \mathrm{~cm}$. The inset plot (c) shows the sound absorption coefficient.

Scanning electron microscope (SEM) images, shown in Fig. 4, have been taken in order to obtain information about the inner structure of P3. Figure 4(b) shows the wall junctions, which resemble Plateau borders ${ }^{36}$ (three and four junctions meet at angles close to $120^{\circ}$ and $109.47^{\circ}$, respectively). From this image, the average wall thickness has been estimated as $h_{w}=0.474 \pm 0.088 \mu \mathrm{m}$. The poly-disperse semiclosed foam-like microstructure of P3 can be identified in Fig. 4(c). A cell size $C_{s}$ is defined as the largest distance between opposite walls in each cell. The mean cell size has been calculated using manual image segmentation and is $C_{s}=47.582 \pm 9.659 \mu \mathrm{m}$. The interscale ratio is therefore given by $\varepsilon_{0}=C_{s} / r_{p}=0.0976$. This confirms the lowpermeability contrast assumption.

\section{Microstructure modeling of expanded perlite}

One can notice from Fig. 4(c) that each cell can be approximated by a polyhedron. The inner geometry of P3 must therefore obey the rules of space-filling packing of polyhedra. ${ }^{37}$ A geometry that satisfies this condition, and 
that related to the Plateau borders law, is the Kelvin foam. ${ }^{38}$ Other possible geometries for modelling foams are the Weaire-Phelan foam, ${ }^{38}$ array of cylinders arranged in a hexagonal lattice, ${ }^{39}$ and array of spherical voids connected by cylindrical pores. ${ }^{40}$ The Weaire-Phelan foam has eight cells in the REV. This low symmetry limits its use. Although an array of cylinders can represent open-cell foams with thin struts, it does not appear to appropriately represent semi-closed foams. Meanwhile, an array of spherical voids connected by cylindrical pores does not obey space-filling rules. The inner geometry of the P3 granules is therefore modeled as a monodisperse array of perforated truncated octahedra. The perforations have been included in an attempt to represent the holes observed in the P3 granules [see Fig. 4(a)]. This implicitly assumes that the fluid phase is connected. The truncated octahedron is an Archimedian solid that has eight regular hexagons and six squares faces $(N=14)$. It corresponds to a flat-faced version of the Kelvin foam. It is generated by subtracting six square pyramids of side $a$ and height $\sqrt{2} a / 2$ from a regular octahedron of side $3 a$ and half height $3 \sqrt{2} a / 2$. A cell size is defined as the distance between the square faces and is given by $C_{s}=2 \sqrt{2} a$. The volume and the area of the truncated octahedron are $V=C_{s}^{3} / 2$ and $A=(3 / 4)(1+2 \sqrt{3}) C_{s}^{2}$. The elementary fluid cell has been built as follows. A truncated octahedron with cell size $C_{s}$ is generated. Then, fourteen cylinders of radius $r_{\mathrm{per}}=\xi C_{s} / 4 \sqrt{2}$ and height $h_{w} / 2$ are located at the center of each face [see Fig. 5(d)]. The parameter $\xi \in[0,1]$ controls the perforation size with respect to half of the smallest face (square of side $a$ ). The microporosity and thermal characteristic length can be calculated as $\phi_{m}=\left(C_{s}^{3}+N \pi r_{p e r}^{2} h_{w}\right) /\left(C_{s}+h_{w} / 2\right)^{3}$ and $3 \Lambda_{m}^{\prime} / 4 C_{s}=(1+$ $\left.\xi^{2} \pi N h_{w} / 32 C_{s}\right) / \psi$, with $\psi=1+2 \sqrt{3}+(\pi N \xi / 3 \sqrt{2})\left(h_{w} / C_{s}\right.$ $-\xi / 4 \sqrt{2})$. The viscous and thermal permeabilities of the microscopic domain have been calculated using the semi-phenomenological models given by Eq. (11). To obtain the values of the model parameters the numerical solution of the static fluid flow [Eq. (5),(6) for $\omega=0$ ], the heat transfer [Eq. (7) for $\omega=0]$, and the high-frequency oscillatory fluid flow (electrical conduction) [Eq. (12)] problems have been performed using the finite element method software COMSOL MULTIPHYSICS. Second-order Lagrangian elements have been used to model the velocity components, whereas the linear elements approximated the pressure field (P2-P1 velocitypressure formulation), as suggested in Ref. 41. An arbitrary reference pressure has been set in one of the vertices of the fluid cell to ensure uniqueness of the solution for the fluid flow problem. ${ }^{42}$ Second-order Lagrangian elements have been used for the temperature and the electric potential for the heat and electrical conduction problems, respectively. In all these problems, periodic boundary conditions were prescribed on the boundaries corresponding to the perforation faces. The convergence of the numerical method has been tested by a careful mesh refining analysis. The microporosity has been set to $\phi_{m}=0.967$ in order to match the measured overall porosity. The diameter of the perforations has been set to half of the smallest face of the truncated octahedron

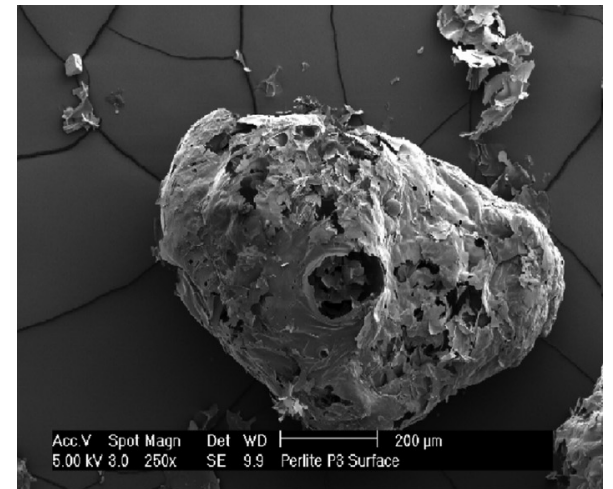

(a)

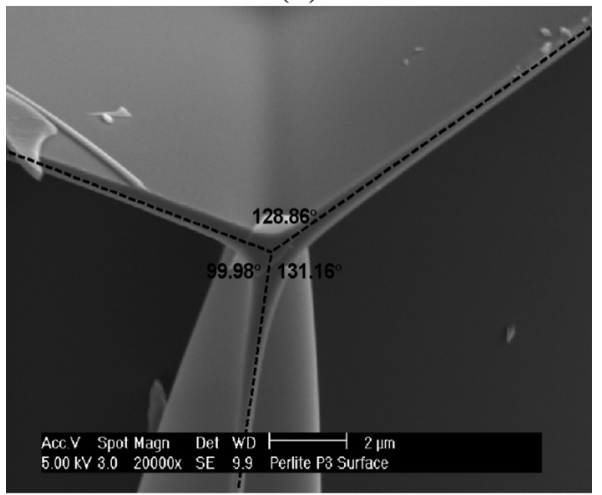

(b)

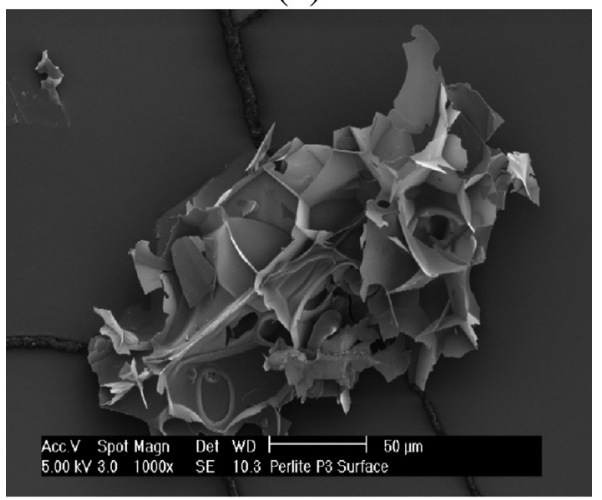

(c)

FIG. 4. Scanning electron microscope images of expanded perlite P3: P3 grain (a), wall junctions (b), and the inner structure (c).

$(q=0.5)$ while the wall thickness to $h_{w}=1.3883 \mu \mathrm{m}$. This value is about three times larger than the one estimated by image processing and has been chosen to match the overall porosity value. The cell size has been, however, set to the measured value. Table I shows the parameters utilized to model the acoustical properties of the array of perforated truncated octahedra. Only results for which the pressure gradient has been applied in the negative $z$-direction are presented here and in the following. These do not differ more than $2.534 \%$ from those obtained when the pressure gradient was applied in the negative $x$ - or $y$-direction. This reflects the quasi-isotropic nature of the microscopic domain.

\section{Comparison with data}

Figure 5 shows the real and imaginary parts of pressure reflection coefficient for a $3-\mathrm{cm}$ hard backed layer of P3. The single porosity model is not capable of reproducing the 


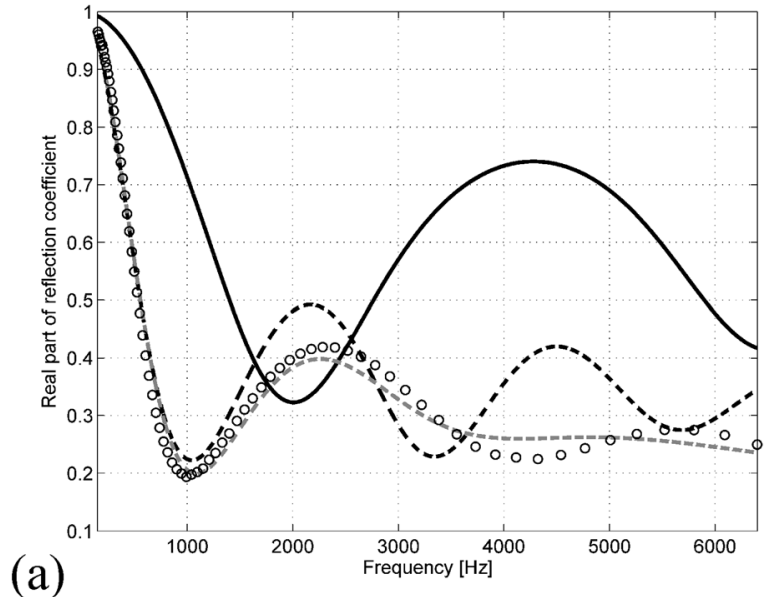

(a)

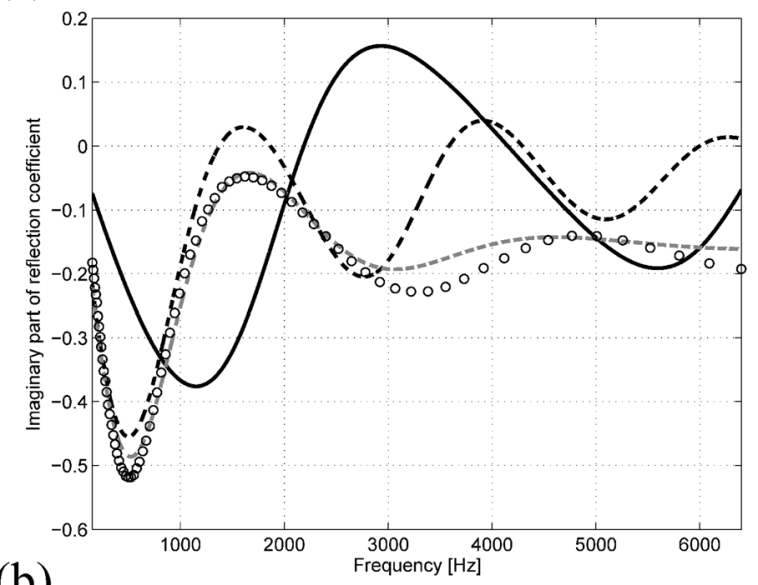

(b)

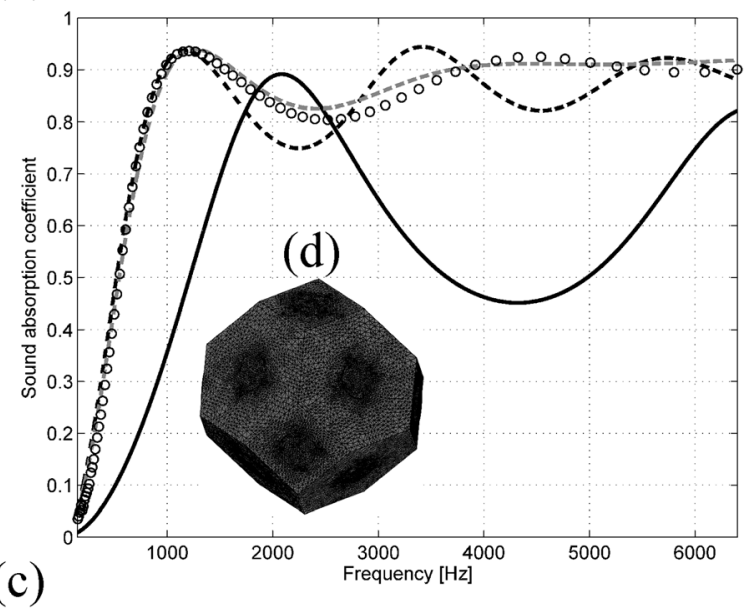

FIG. 5. Real (a) and imaginary (b) parts of the reflection coefficient and sound absorption coefficient (c) of a 3-cm hard-backed layer of expanded perlite P3. Circles—data. Continuous black line-analytical single porosity model. Dashed black line-hybrid double porosity model. Dashed gray line-fitted analytical double porosity model. (d) geometry and mesh of the elementary fluid cell.

data over the whole range of frequency. A reasonably good agreement is found between the data and the hybrid model. The following simplifications used in the modeling could explain the observed differences between data and the predictions. The analytical model for the mesodomain assumes that the particles are spherical and monodisperse while P3 is clearly a polydisperse granular material with nonspherical grains. The same argument applies to the microscopic domain as it has been modeled as a monodisperse array of
TABLE I. Parameters for the microdomain of expanded perlite P3.

\begin{tabular}{lcccccc}
\hline \hline $10^{3} k_{0 m} / C_{s}^{2}$ & $10^{3} k_{0 m}^{\prime} / C_{s}^{2}$ & $\alpha_{0 m}$ & $\alpha_{0 m}^{\prime}$ & $\alpha_{\infty m}$ & $\Lambda_{m} / C_{s}$ & $\Lambda_{m}^{\prime} / C_{s}$ \\
\hline 0.7051 & 14.844 & 2.3519 & 1.45 & 2.0431 & 0.1066 & 0.3239 \\
\hline \hline
\end{tabular}

perforated truncated octahedra. On the other hand, the parallel fluid flow model used to calculate the viscous permeability might not be a realistic assumption as this expression only holds for mesoscopic geometry with straight parallel cylindrical or slit pores. ${ }^{22}$ A more general approach may be based on solving the set of equations A1 in Ref. 22. A better agreement can be obtained by fitting the analytical double porosity model to the data. This procedure has been implemented by using the differential evolution algorithm. ${ }^{43}$ The fitted values for the micropore and particle radii and the porosities are $r_{m}=28.055 \mu \mathrm{m}, \phi_{m}=0.999, r_{p}=0.599 \mathrm{~mm}$, and $\phi_{p}=0.2871$. This results in an overall porosity of $\phi_{d b}=0.997$, which is $2.26 \%$ larger than the measured value. The fitted micropore diameter appears to be comparable to the measured average cell size plus its standard deviation while the fitted particle diameter corresponds to the upper limit of the nominal 80\%-band grain size. Although a much closer agreement is obtained, it is recognized that a staggered array of circular pores does not correspond to the actual microdomain geometry of P3. The disagreement between the data and the predictions is not very pronounced for sound absorption coefficient as is shown in Fig. 5(d). The general trend is that a double porosity low-permeability contrast granular material presents larger sound absorption at low frequencies compared to a solid-grain material with the same mesoscopic characteristics at reduced weight.

\section{Double porosity granular materials with high-permeability contrast}

\section{Characterization}

Two samples of granular activated carbon made of coal have been used as examples of double porosity granular

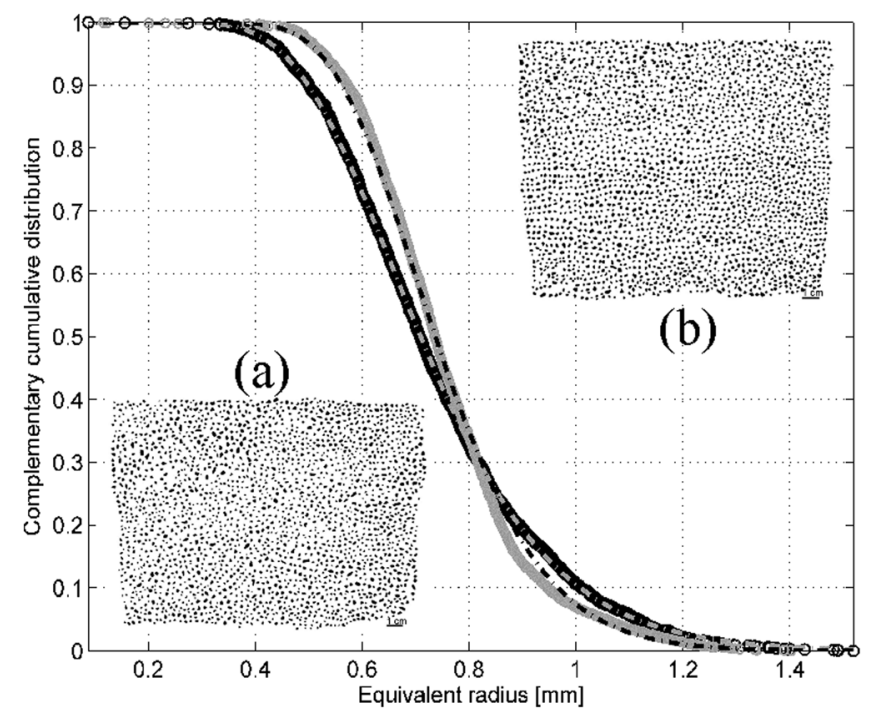

FIG. 6. Complementary cumulative distributions of the equivalent particle radius for the activated carbon samples. Gray circles: data for SRD71. Black circles: data for SRD75. Dashed dotted lines-fitted distributions. The inset plots show the processed images. (a) SRD75 and (b) SRD71. 
TABLE II. Properties of activated carbon samples.

\begin{tabular}{lccccc}
\hline \hline Sample & $\begin{array}{c}\text { Bulk density } \\
\rho_{b}\left(\mathrm{~g} / \mathrm{cm}^{3}\right)\end{array}$ & $\begin{array}{c}\text { N2 surface } \\
\text { area }\left(\mathrm{m}^{2} / \mathrm{g}\right)\end{array}$ & $\begin{array}{c}\text { CO2 surface } \\
\text { Area }\left(\mathrm{m}^{2} / \mathrm{g}\right)\end{array}$ & $\begin{array}{c}\text { Nanopore volume } \\
V_{n}(0-2 \mathrm{~nm})\left(\mathrm{cm}^{3} / \mathrm{g}\right)\end{array}$ & $\begin{array}{c}\text { Everall } \\
\text { porosity } \phi_{d b}\end{array}$ \\
\hline SRD71 & 0.566 & 665 & 371 & 0.283 & 0.7427 \\
particle radius $\left(\mathrm{mm}^{2}\right)$ & 0.774 & 0.8477 \\
\hline \hline
\end{tabular}

materials with high permeability contrast. Activated carbon is normally manufactured by carbonizing raw material followed by an activation process by either oxidization of $\mathrm{CO}_{2}$ or steam. This process creates a hierarchical porosity ranging from nanometer to micrometer size pores within the granules. Activated carbon is commonly used in filtration and purification processes due to its remarkably large surface area and sorption capacity. The samples will be referred to as SRD71 and SRD75 in the following. Optical granulometry has been applied to these two samples. The image processing is the same as the one implemented for the lead shot sample. Figure 6 shows the complementary cumulative distribution of the equivalent particle radius for SRD71 and SRD75. The inset plots show the processed images. The number of particles considered for SRD71 is 2439 and the average mass per particle is $0.8319 \mathrm{mg}$. In the case of SRD75, these correspond to 2208 and $0.4792 \mathrm{mg}$, respectively. For both samples, the equivalent particle radius follows a lognormal distribution, $f(t \mid \mu, \theta)=(1 / t \theta \sqrt{2 \pi}) \exp \left(-(\ln (t)-\mu)^{2} / 2 \theta^{2}\right)$. The parameters of the fitted distribution for SRD71 are $\mu=-7.2129$ and $\theta=0.2112$ while those for SRD75 are $\mu=-7.2513$ and $\theta=0.2741$. These two samples are very similar in terms of mesoscopic characteristics. The difference in their inner structure is significant as the sample SRD75 is more porous than SRD71. This can be deduced from the average mass per particle and more explicitly from Table II where the bulk density, pore surface area, nanopore volume, and the estimated overall porosity are presented along with the expected value and standard deviations (in parenthesis) of the equivalent particle radius for SRD71 and SRD75. The tabulated density of carbon black $\rho_{c}=2.2 \mathrm{~g} /$ $\mathrm{cm}^{3}$ has been used to estimate the overall porosity.

In double porosity materials with high-permeability contrast the fluid flow is not affected by the micropores. To justify the applicability of this approximation, flow resistivities of the two activated carbon samples have been measured. The procedure is detailed in the standard ${ }^{44}$ BS EN 29053:1993. Table III presents the flow resistivity for SRD71 and SR75 for a layer of $4 \mathrm{~cm}$ and two samples with layer thicknesses of $2 \mathrm{~cm}$. The other columns show a theoretical estimation of mesoporosity that matches the measured flow resistivity assuming identical spheres with their radii given by the expected values of the equivalent particle radius distribution (last column of Table II). The flow resistivity was calculated as $\sigma_{0 p}=\eta / k_{0 p}$, where the static viscous permeability of the spheres packing is given by Eq. (21). The observed variability in flow resistivity is $6.85 \%$ for SRD71 and $6.14 \%$ for SRD75. This can be explained with an average variability in mesoporosity of 1.77 and $1.62 \%$, respectively. The average flow resistivity of SRD75 is slightly larger than that of SRD71 due to its higher dust content. Consequently, its mesoporosity is smaller. The flow resistivity and the mesoporosity can be considered thickness independent and not significantly affected by the activated carbon packing conditions. The fact that the two samples with very similar mesoscopic characteristics and different microscopic characteristics have close flow resistivity values justifies the high-permeability contrast assumption. These results also allow estimating the microporosity as $\phi_{m}=\left(\phi_{d b}-\phi_{p}\right) /\left(1-\phi_{p}\right)$.

\section{Comparison with data}

The data of pressure reflection coefficient for a rigidlybacked 2-cm layer of SRD71 along with the model predictions is presented in Figs. 7(a) and 7(b). The particle radius and the mesoporosity values are given in Tables II and III. The microporosity $\phi_{m}=0.628$ has been calculated using mesoporosity and overall porosity values. The micropore radius has been set to $r_{m}=0.7125 \mu \mathrm{m}$. This has been calculated through a best fitting routine using the differential evolution algorithm. ${ }^{43}$ This value correlates well with the mean size of the macropores (or transport pores) commonly found in activated carbon ${ }^{45}$ and provides an interscale ratio of $\varepsilon_{0}=r_{m} / r_{p}=0.9455 \times 10^{-3}$, which is in line with the high-permeability contrast assumption. On the other hand, the static viscous permeability including rarefaction effects is given by ${ }^{46} k_{0 m}\left(K_{n}\right)=\phi_{m} r_{m}^{2}\left(1+4 K_{n}\right) / 8$, where the Knudsen number is defined as ${ }^{46} K_{n}=l_{\text {mean }} / r_{m}$. Considering that the molecular mean free path $l_{\text {mean }}$ is approximately $60 \mathrm{~nm}$ at normal conditions, ${ }^{47}$ the calculation of the static viscous

TABLE III. Flow resistivity data and mesoporosity estimation for activated carbon.

\begin{tabular}{lcccc}
\hline \hline & \multicolumn{2}{c}{ Flow resistivity $\left(\mathrm{kPa} \mathrm{s} / \mathrm{m}^{2}\right)$} & \multicolumn{2}{c}{ Mesoporosity $\phi_{p}$} \\
\cline { 2 - 3 } & SRD71 & SRD75 & SRD71 & SRD75 \\
\hline $\mathrm{A}(d=4 \mathrm{~cm})$ & 20.041 & 23.0667 & 0.312 & 0.305 \\
$\mathrm{~B}(d=2 \mathrm{~cm})$ & 20.4436 & 24.6232 & 0.302 & 0.299 \\
$\mathrm{C}(d=2 \mathrm{~cm})$ & 22.7196 & 26.0870 & 0.311 & 0.295 \\
Average & $21.068 \pm 1.444$ & $24.5923 \pm 1.5104$ & $0.3083 \pm 0.0055$ & $0.2997 \pm 0.005$ \\
\hline \hline
\end{tabular}


permeability would differ by $33.6 \%$. This seemingly large difference does not significantly modify the predictions of the acoustical quantities in the audible frequency range [see, for example, Eq. (64) in Ref. 47]. The double porosity model provides much closer agreement with the data than the single porosity model. However, its accuracy is affected by the fact that only a limited amount of information about the material microstructure was available. Figures 7(c) and 7(d) present the same information as Figs. 7(a) and 7(b) but for the sample SRD75. The calculated microporosity value is $\phi_{m}=0.7825$ while the fitted micropore radius is $r_{m}=0.9881 \mu \mathrm{m}$. This value gives an interscale ratio of $\varepsilon_{0}=1.3419 \times 10^{-3}$. The agreement between the double porosity model and the data is better than that for the single porosity model. This is emphasized in Fig. 8 where absorption coefficient data for both samples is presented along with predictions of the single porosity and double porosity models. The single porosity model predictions are close due to similar mesoscopic characteristics of the two samples. The double porosity models predictions of absorption coefficient are also close at low frequencies. This is expected since the static flow resistivity values of the samples are close.

To provide an insight into the low-frequency behavior, the dynamic bulk moduli for both activated carbon samples and the lead shots have been deduced from the low-frequency characteristic impedance and wave number measured using the two-thickness method ${ }^{48}$ as $K_{d b}=\omega Z_{d b}^{c} / q_{d b}^{c}$. It is shown in Fig. 9 that the normalized static value of the dynamic bulk modulus of the lead shots is accurately predicted and given by $1 / \phi_{p} \approx 1 / 0.3905=2.5608$. For SRD71 the predicted value is $1 / 0.7427=1.3464$ while for SRD75 is $1 / 0.8477=1.1797$. By extrapolating the real part of the dynamic bulk modulus to zero frequency one can conclude that the static limit is 0.5777 for SRD71 and 0.4274 for SRD75. These values cannot be explained by the proposed model and suggest that the theory may need to be extended to account for physical processes that modify the static bulk modulus. This quantity is modified when the finite heat capacity of the solid is taken into account. ${ }^{4}$ According to Eq. (B10) in Ref. 4, the static bulk modulus is given by $\phi K(\omega \rightarrow 0) / P_{0}=(1+h) /(1+h / \gamma)$, where $h$ is the ratio of the heat capacity of air to that of the solid. For the activated carbon samples $h=O\left(10^{-3}\right)$ and the experimental trend cannot therefore be explained. Thermal slip effects modify the dynamic bulk modulus but not its static value ${ }^{46,47}$ Mass transfer processes also alter the dynamic bulk modulus. For example, Raspet et al. ${ }^{49}$ have studied the sound attenuation in rigid cylindrical pores filled with air and saturated water vapor accounting for the mass transfer of vapor from the wet tube wall. According to Eq. (47) in Ref. 49 and equation of state, the static bulk modulus for an array of cylindrical pores with wet walls can be written in terms of the ratio between the partial pressure of air $p_{1}$ and that of water vapor $p_{2}$ as $\phi K(\omega \rightarrow 0) / P_{0}=1 /\left(1+p_{2} / p_{1}\right)$. However, this ratio is small at normal conditions ${ }^{49}$ and might not be able to explain the measured activated carbon static bulk modulus value.

The presence of adsorbed films of water at the contact point of the granules has been identified as the main cause of both dampening structure-borne sound and shifting the resonant frequency of a bar filled with tungsten particles
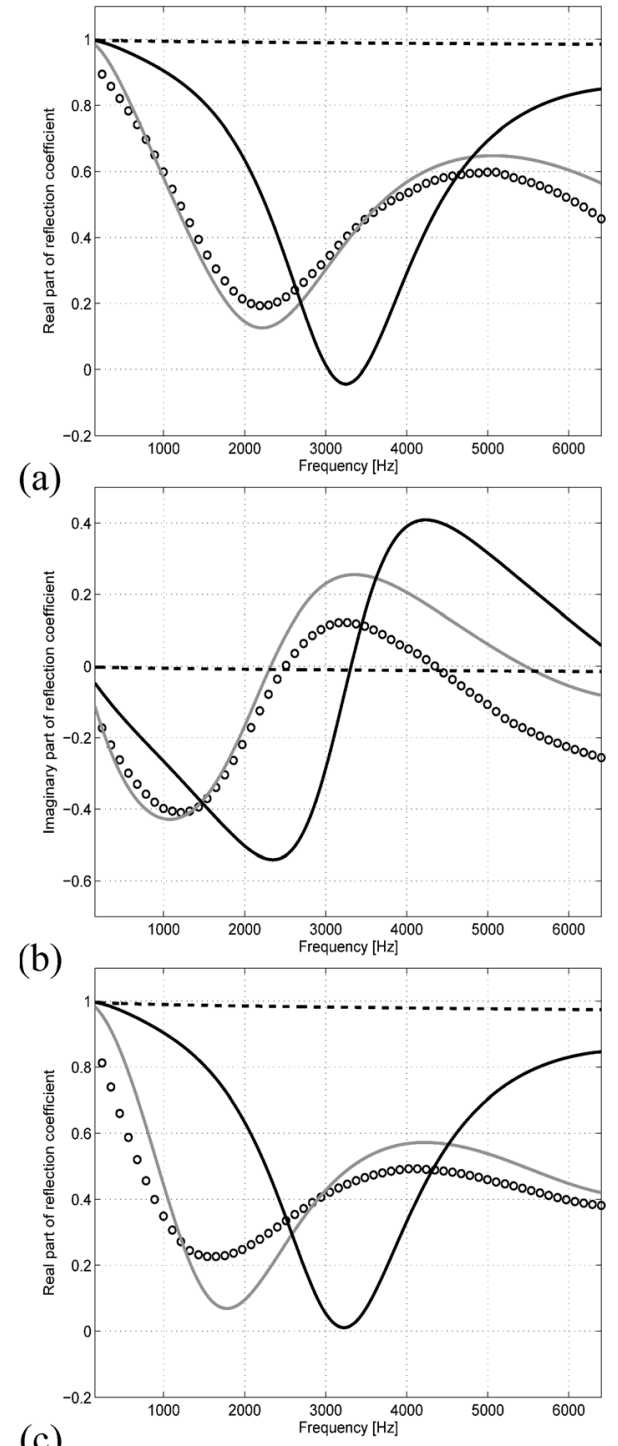

(c)

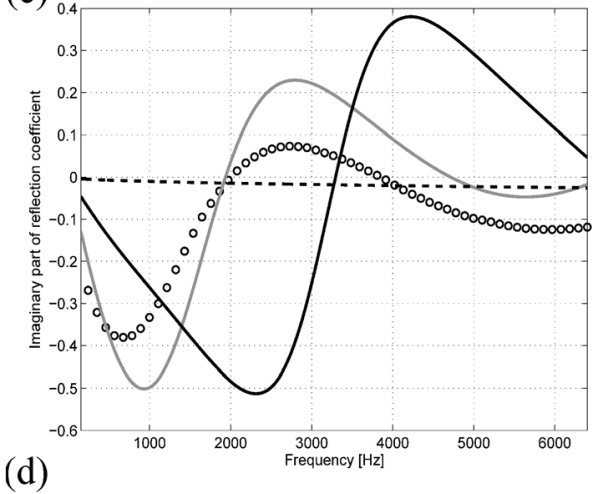

FIG. 7. Real (a) and imaginary (b) parts of the reflection coefficient of a 2 -cm hard-backed layer of SRD71. Circles-data. Continuous gray linedouble porosity model. Continuous black line - single porosity model for a packing of solid particles [Eqs. (14) and (22)]. Dashed black line-single porosity model for the microscopic domain alone [Eqs. (23)]. The same information but for SRD75 is shown in (c) and (d).

towards lower frequencies. ${ }^{50}$ A similar shift has been documented in loudspeakers ${ }^{51}$ partially filled and resonators ${ }^{52}$ fully filled with activated carbon. This effect has been however attributed to sorption processes that occur in the small pores within the grains. The effect of sorption on sound 


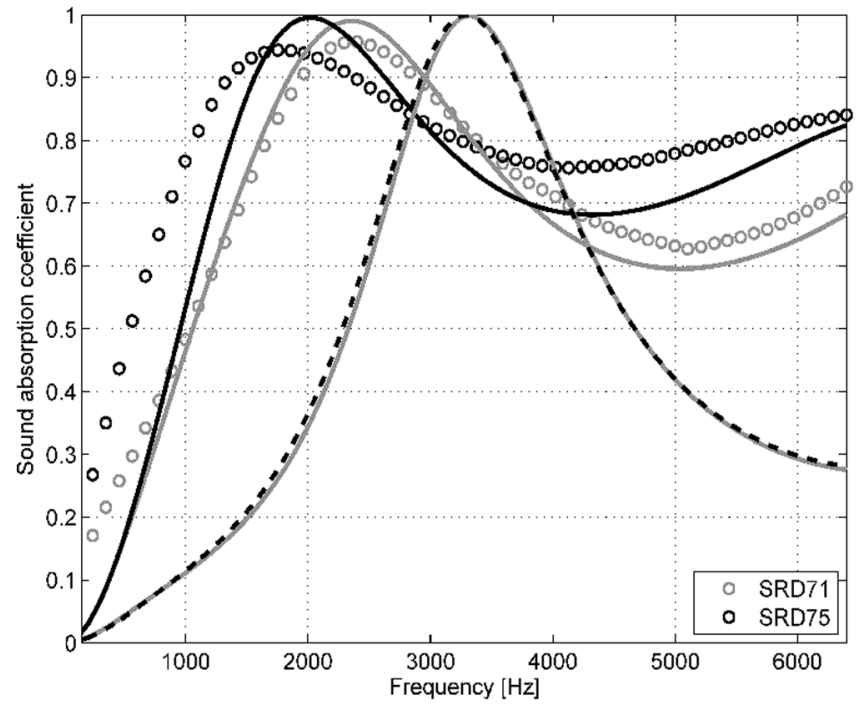

FIG. 8. Sound absorption coefficients of 2-cm hard-backed layers of SRD71 (gray circles) and SRD75 (black circles). Continuous lines-double porosity model predictions. Dashed lines-single porosity model predictions for packing of solid particles.

attenuation in straight cylindrical pores has been studied by Mellow et al. ${ }^{53}$ who approximated the sorption dynamics by a Langmuir isotherm model. From Eq. (39) in Ref. 53, the static bulk modulus can be deduced as $\phi K(\omega \rightarrow 0) / P_{0}=1 /\left(1+P_{0} k_{a} k_{d} \rho_{N} /\left(k_{a} P_{0}+k_{d}\right)^{2} \rho_{0}\right)$, where $k_{a}$ and $k_{d}$ are the adsorption and desorption constants and $\rho_{N}=2 \sigma_{s} / r_{n}$ is the maximum adsorbed density. Here, the surface mass density that can be accommodated is denoted as $\sigma_{s}$ while the pore radius as $r_{n}$. This expression might be able to explain the general trends in the behaviour of the bulk modulus of activated carbon. However, the Langmuir model is a poor approximation to the sorption characteristics of activated carbon. A more comprehensive approach based for instance on the Freundlich adsorption isotherm model ${ }^{45}$

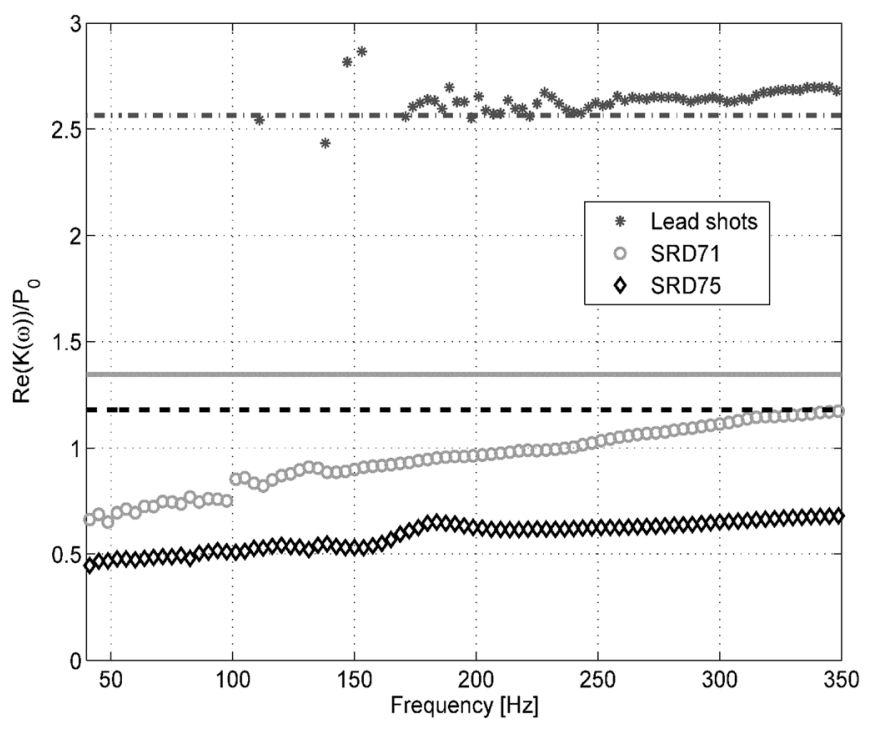

FIG. 9. Normalized measured real part of the low-frequency dynamic bulk modulus for SRD75 (black diamonds), SRD71 (gray circles), and lead shots (gray asterisks). The lines represent the predicted static bulk moduli $1 / \phi_{p, d b}$. might be necessary. It should also be noted that the friction caused by the particle vibration has been suggested to increase the low-frequency sound absorption in granular materials. ${ }^{54}$ Particle motion, mass transfer and sorption processes are likely to be occurring during sound propagation in activated carbon and need to be included into a more general theory. This theory could also include an additional porosity scale at the nanometre level, which seems to be the case that would better describe the acoustical properties of activated carbon. This idea is motivated by the larger nanoporosity of SRD75 $\left(\phi_{n}=\rho_{b} V_{n}=0.2593\right)$ compared to that of SRD71 $\left(\phi_{n}=0.1602\right)$ (see Table II).

\section{CONCLUSIONS}

Double porosity materials have been intensively studied in the past decade. It has been proven that they can achieve larger low frequency sound absorption compared to single porosity materials. However, granular materials with porous particles, which are commonly used in building and chemical industries, have received relatively little attention. These materials come with a large variety of particle and pore sizes which allows low as well as high permeability contrast. In this paper, the acoustical properties of double porosity granular materials have been studied both theoretically and experimentally. The existing self-consistent model for a packing of identical solid spheres has been extended to allow for particle porosity. Expanded perlite has been used as an example of a granular material with low permeability contrast. High permeability contrast has been achieved in samples of activated carbon. It has been demonstrated that packings of porous particles provide much improved low frequency sound absorption compared to that of solid particles with the same mesoscopic characteristics at reduced weight. This makes these materials potentially attractive for acoustic applications. It has also been found that the low frequency properties of activated carbon cannot be completely explained by their double porosity structure as the measured static values of the bulk moduli are lower than those predicted by the theory of sound propagation in double porosity materials. This might be an indication of mass transfer and sorption processes happening in smaller pores. The investigation of these effects and their use in designing new acoustic materials are interesting topics for future research.

\section{ACKNOWLEDGMENTS}

R.V. gratefully acknowledges the ORSAS award and the University of Salford Research Studentship. The authors are grateful to Chemviron and William Sinclair Holdings PLC for kindly supplying the samples investigated in this paper.

${ }^{1}$ J. F. Allard and N. Atalla, Propagation of Sound in Porous Media: Modeling Sound Absorbing Materials (Wiley, New York, 2009), Chaps. 2-5.

${ }^{2}$ M.A, Biot, "Theory of propagation of elastic waves in a fluid saturated porous solid. I. Low-frequency range," J. Acoust. Soc. Am. 28, 168-178 (1956); M. A. Biot, "Theory of propagation of elastic waves in a fluid saturated solid. II. High-frequency range,” ibid. 28, 179-191 (1956).

${ }^{3}$ D. L. Johnson, J. Koplik, R. Dashen,"Theory of dynamic permeability and tortuosity in fluid-saturated porous media," J. Fluid Mech. 176, 379-402 (1987).

${ }^{4}$ D. Lafarge, P. Lemarinier, J. F. Allard, and V. Tarnow, "Dynamic compressibility of air in porous structures at audible frequencies," J. Acoust. Soc. Am. 102(4), 1995-2006 (1997). 
${ }^{5}$ S. R. Pride, F. D. Morgan, and A.F. Gangi, "Drag forces of porousmedium acoustics," Phys. Rev. B 47, 4964-4975 (1993).

${ }^{6} \mathrm{D}$. Lafarge, "Propagation du son dans les matériaux poreux à structure rigide saturés par un fluide viscothermique," Ph.D. thesis, Université du Maine, Le Mans, France (1993) (Sound propagation in rigid porous media saturated by a viscothermal fluid).

${ }^{7}$ J. L. Auriault, L. Borne, and R. Chambon, "Dynamics of porous saturated media, checking of the generalized law of Darcy," J. Acoust. Soc. Am. 77(5), 1641-1650 (1985).

${ }^{8}$ J. F. Allard, M. Henry, J. Tizianel, L. Kelders, and W. Lauriks, "Sound propagation in air-saturated random packings of beads," J. Acoust. Soc. Am. 104, 2004-2007 (1998).

${ }^{9}$ F. Asdrubali and K. V. Horoshenkov, "The acoustic properties of expanded clay granulates," Building Acoust. 9(2), 85-98 (2002).

${ }^{10} \mathrm{~V}$. V. Voronina and K.V. Horoshenkov, "A new empirical model for the acoustic properties of loose granular media," Appl. Acoust. 64, 415-432 (2004).

${ }^{11}$ A. M. Chapman and J. J. L. Higdon, "Oscillatory Stokes flow in periodic porous media," Phys. Fluids 4, 2099-2116 (1992).

${ }^{12} \mathrm{O}$. Umnova, K. Attenborough, and K. M. Li, "Cell model calculations of dynamic drag parameters in packings of spheres," J. Acoust. Soc. Am. 107, 313-318 (2000).

${ }^{13} \mathrm{O}$. Umnova, K. Attenborough, and K. M. Li, "A cell model for the acoustical properties of packings of spheres," Acta Acust. 87, 226-235 (2001).

${ }^{14}$ E. Sanchez-Palencia, Nonhomogeneous Media and Vibration Theory, Lecture Notes in Physics, edited by J. Ehlers, K. Hepp, R. Kippenhahn, and J. Zittartz (Springer-Verlag, Berlin,1980).

${ }^{15}$ J. L. Auriault, C. Boutin, and C. Geindreau, Homogenization of Coupled Phenomena in Heterogenous Media (Wiley, London, 2009).

${ }^{16} \mathrm{~S}$. Gasser, F. Paun, and Y. Brechet, "Absorptive properties of rigid porous media: Application to face centered cubic sphere packing," J. Acoust. Soc. Am. 117(4), 2090-2099 (2005).

${ }^{17}$ C.-Y. Lee, M. J. Leamy, and J. H. Nadler, "Acoustic absorption calculation in irreducible porous media: A unified computational approach," J. Acoust. Soc. Am. 126(4), 1862-1870 (2009).

${ }^{18} \mathrm{C}$. Boutin and C. Geindreau, "Estimates and bounds of dynamic permeability of granular media," J. Acoust. Soc. Am. 124(6), 3576-3593 (2008).

${ }^{19} \mathrm{C}$. Boutin and C. Geindreau, "Periodic homogenization and consistent estimates of transport parameters through sphere and polyhedron packings in the whole porosity range," Phys. Rev. E 82, 036313 (2010).

${ }^{20} \mathrm{~J}$. L. Auriault and C. Boutin, "Deformable porous media with double porosity III: Acoustics," Transp. Porous Media 14, 143-162 (1994).

${ }^{21}$ C. Boutin, P. Royer, and J. L. Auriault, "Acoustic absorption of porous surfacing with dual porosity,” Int. J. Solids Struct. 35, 4709-4737 (1998).

${ }^{22} \mathrm{X}$. Olny and C. Boutin, "Acoustic wave propagation in double porosity media," J. Acoust. Soc. Am 114(1), 73-89 (2003).

${ }^{23}$ G. Pispola, K. V. Horoshenkov, and A. Khan, "Comparison of two modeling approaches for highly heterogeneous porous media," J. Acoust. Soc. Am. 121(2), 961-966 (2007).

${ }^{24}$ N. Atalla, F. Sgard, X. Olny, and R. Panneton, "Acoustic absorption of macro-perforated porous materials," J. Sound. Vibr. 243(4), 659-678 (2001).

${ }^{25} \mathrm{E}$. Gourdon and M. Seppi, "Extension of double porosity model to porous materials containing specific porous inclusions," Acta Acust. United Acust. 96(2), 275-291 (2010)

${ }^{26}$ J. Chastanet, P. Royer, and J.-L. Auriault, “Acoustics with wall-slip flow of gas saturated porous media,” Mech. Res. Commun. 31, 277-286 (2004).

${ }^{27}$ J. Chastanet, P. Royer and J.-L. Auriault,"Flow of low pressure gas through dual-porosity media," Transp. Porous Media 66(3), 457-479 (2007).

${ }^{28} \mathrm{R}$. Venegas and O. Umnova, "Numerical modelling of sound absorptive properties of double porosity granular materials," in Proceedings of Comsol Conference 2010, Paris, France, 2010, Paper No. 8303, http:// www.comsol.com/papers/8303 (Last viewed April 14, 2011).

${ }^{29}$ R. J. S. Brown, "Connection between formation factor for electricalresistivity and fluid-solid coupling factor in Biot equations for acoustic waves in fluid-filled porous media," Geophysics 45, 1269-1275 (1980).
${ }^{30}$ Y. Champoux and J. F. Allard, "Dynamic tortuosity and bulk modulus in air-saturated porous media,” J. Appl. Phys. 70(4), 1975-1979 (1991).

${ }^{31}$ M. Abramowitz and I. A. Stegun, Handbook of Mathematical Functions With Formulas, Graphs, and Mathematical Tables (Dover, New York, 1972), Chap. 10

${ }^{32}$ ISO 10534-2:2001, "Acoustics-Determination of sound absorption coefficient and impedance in impedance tubes-Part 2: Transfer-function method, 2001.

${ }^{33}$ J. Serra. Image Analysis and Mathematical Morphology (Academic Press, London, 1982), Chap. 11.

${ }^{34}$ W. Man, A. Donev, F. Stillinger, M. Sullivan, W. Russel, D. Heeger, S. Inati, S. Torquato, and P. M. Chaikin, "Experiments on random packings of ellipsoids," Phys. Rev. Lett. 94, 198001 (2005).

${ }^{35}$ C. Song, P. Wang, and H.A. Makse, "A phase diagram for jammed matter," Nature 453, 629-632 (2008).

${ }^{36}$ J. Plateau, Statique Expérimentale et Théorique des Liquides Soumis aux Seules Forces Moléculaires (Gauthier, Paris, 1873), Vol. 1, Chap. 5.

${ }^{37}$ N.J. Mills, Polymer Foams Handbook: Engineering and Biomechanics, Applications and Design Guide (Butterworth-Heinemann, London, 2007), Chap. 1.

${ }^{38}$ D. L. Weaire, The Kelvin Problem: Foam Structures of Minimal Surface Area (Taylor and Francis, London, 2000).

${ }^{39}$ C. Perrot, F. Chevillotte, and R. Panneton, "Dynamic viscous permeability of an open-cell aluminum foam: Computations versus experiments," J. Appl. Phys. 103, 024909 (2008)

${ }^{40}$ F. Chevillotte, C. Perrot, and R. Panneton,"Microstructure based model for sound absorption predictions of perforated closed-cell metallic foams," J. Acoust. Soc. Am.128(4), 1766-1776 (2010).

${ }^{41}$ T. J. Chung, Computational Fluid Dynamics (Cambridge University Press, Cambridge, 2002), Chaps. 10 and 12.

${ }^{42}$ W. Zimmerman, Process Modelling and Simulation with Finite Element Methods, Series on Stability (World Scientific, Singapore, 2004), p. 182.

${ }^{43}$ K. Price, R. Storn, and J. Lampinen, Differential Evolution: A Practical Approach to Global Optimization (Springer-Verlag, Berlin, 2005); see also http://www.icsi.berkeley.edu/storn/code.html (Last viewed March 31, 2011).

${ }^{44}$ EN 29053:1993, "Acoustics. Materials for acoustical applications. Determination of airflow resistance," 1993.

${ }^{45}$ D. D. Do, Adsorption Analysis: Equilibria and Kinetics (Imperial College Press, London, 1998), pp. 5 and 50.

${ }^{46}$ V. F. Kozlov, A. V. Fedorov, and N. D. Malmuth, "Acoustic properties of rarefied gases inside pores of simple geometries," J. Acoust. Soc. Am. 117, 3402-3411 (2005)

${ }^{47}$ O. Umnova, D. Tsiklauri, and R. Venegas, "Effect of boundary slip on the acoustical properties of microfibrous materials," J. Acoust. Soc. Am. 126(4), 1850-1861 (2009).

${ }^{48}$ C. D. Smith and T. L. Parrott, "Comparison of three methods for measuring acoustic properties of bulk materials," J. Acoust. Soc. Am. 74(5), $1577-1582$ (1983)

${ }^{49}$ R. Raspet, C. J. Hickey, and J. M. Sabatier, "The effect of evaporationcondensation on sound propagation in cylindrical tubes using the low reduced frequency approximation," J. Acoust. Soc. Am. 105(1), 65-73 (1999).

${ }^{50}$ J. Valenza, C.-J. Hsu, R. Ingale, N. Gland, H. A. Makse, and D. L. Johnson, "Dynamic effective mass of granular media and the attenuation of structure-borne sound," Phys. Rev. E 80, 051304 (2009).

${ }^{51}$ J.R. Wright, "The virtual loudspeaker cabinet," J. Audio Eng. Soc. 51(4), 244-247 (2003)

${ }^{52} \mathrm{~F}$. Bechwati, Acoustics of activated carbon, Ph.D. thesis, University of Salford, Salford, UK, 2008.

${ }^{53}$ T. J. Mellow, O. Umnova, K. Drossos, K. Holland, A. Flewitt, and L. Kärkkäinen, "On the adsorption-desorption relaxation time of carbon in very narrow ducts," in Proceedings of Acoustics 08, CD-ROM 01, pp. 795-800, Paris, France, 2008, available at http://intellagence.eu.com/acoustics2008/ acoustics2008/cd1/data/articles/000871.pdf (Last viewed April 14, 2011)

${ }^{54}$ Y. Okudaira, Y. Kurihara, H. Ando, M. Satoh, and K. Miyanami, "Sound absorption measurements for evaluating dynamic physical properties of a powder bed,” Powder Technol. 77(1), 39-48 (1993). 